\title{
The effect of group size on group information transmitted
}

\section{FREDERICK W. HUFF AND THOMAS P. PIANTIANIDA, UNIVERSITY OF SOUTH FLORIDA, Tampa, Florida 33620}

Group information transmitted was computed for different size groups and it was found that as group size increased information transmitted decreased. The use of groups as a basis for computing uncertainty was discussed.

It is usual in using information theory to compute information transmitted $\left(\mathrm{T}_{\mathrm{x}: \mathrm{y}}\right)$ for an individual. Then, if interested in comparing information transmitted under several conditions, groups of individuals would be set up, $T_{x ; y}$ computed for each individual, and group averages obtained. In this way the amount of information of any group would be the average of the information transmitted within each individual in the group. Recently, information or uncertainty has been computed within groups. In this situation $T_{x: y}$ is, most simply, a function of (1) stimulus uncertainty $\left(\mathrm{H}_{x}\right),(2)$ response uncertainty $\left(\mathrm{H}_{y}\right)$, minus (3) an estimate of interiudge agreement or shared constraint $\left(\mathrm{H}_{\mathrm{x} ; \mathrm{y}}\right)$. Bieri, Atkins, Briar, Leaman, Miller, \& Tripodi $(1966$, p. 83$)$ used the group channel capacity ( $T_{\mathbf{x}}$; computed on the basis of pooled Ss' responses), and indicated that since interjudge reliability was usually less than intrajudge reliability $T_{x ; y}$ would be higher for individuals than groups. However, they did not explore this or investigate the effect (if any) of variations in the size of the group on group $T_{x ; y}$.

Furthermore, in multivariate uncertainty analyses, $T_{x: y}$ might be computed by averaging across individuals on some variables while not on others. Take for example an experiment by Clement (1964). Three variables were involved, stimuli, response categories, and Ss. $T_{x ; y}$ for response categories was obtained by collapsing across $S s$, which in effect, was calculating a group $T_{x: y}$ rather than one based on individuals. This is by way of saying that group $T_{x ; y}$ is often used, even when not intended. It is obvious that if there is some systematic relationship between the size of the group and group $T_{x: y}$ it should be known, even if only to deter researchers from using groups of unequal $\mathrm{N}$, in which case group size effects would be confounded with experimental effects.

The following experiment was designed to explore the effect of variations in group size on group $T_{x: y}$. From some preliminary data it was believed that as the number of individuals constituting a group increased group $T_{x ; y}$ would decrease.

Subjects. Fifty-five undergraduates at the University of South Florida served as Ss. By using a table of random numbers, they were randomly assigned to 10 groups, with a different number of Ss in each group. Group 1 had one $S$, and thus is really no group at all; Group 2 had two; Group 3, three; Group 4, four; Group 5, five; Group 6, six; Group 7, seven; Group 8, ten; Group 9, 12; and Group 10, 14 Ss. All 55 Ss were then pooled again and the above procedure repeated, so that now there were two groups of each size. This was done eight more times or a total of 10 times. As a result 90 groups were assembled, 10 for each sample size.

Procedure. All $55 \mathrm{Ss}$ together were presented two classes of stimuli, each class containing six separate stimuli, and asked to judge where these stimuli fell on six point scales. The first class was six person-object stimuli where each person-object was represented by five statements. These statements were chosen from the Maudsley Personality Inventory 1 because they were found to reflect a person's position on the dimension of neuroticism or adjustment. The ratio of non-neurotic to neurotic statements varied for each of the six person objects. For example, all of the statements for Person A were loaded high on neuroticism. Person B, however, had only nonneurotic statements.

The group of Ss were presented (by use of overhead projector) each of the six person-objects in a random order. They were told that the five statements were statements made by a person, e.g., Person D, and then each of the five statements were read aloud to the Ss. They were then asked to mark on a six-point scale of adjustment where they thought this individual might be. This was repeated for the five other person-objects. The rating scale provided was a 6 by 6 grid with the six person-objects indicated by the columns and the six response categories by the rows. Verbal response categories or the row definitions were provided, e.g., highly adjusted, maladjusted, etc.

The second class of stimuli presented the Ss were six lights varying in degree of redness (and to a certain extent, but unsystematically, luminance). These lights were projected by means of a Kodak Carousal projector in a dimly illuminated room. The task was for each $S$ to indicate degree of redness on a six-point scale with verbal labels defining these points, e.g., red, reddish, orange, etc.

In sum, 55 Ss were given 12 stimuli, six from two different classes, and asked to rate each on a six-point scale, the scales being appropriate to the stimulus class presented, either adjustment or redness.

Results. An uncertainty analysis was performed on the data from each of the 90 groups and for all the Ss combined into one group. This was done in the following manner. A 6 by 6 matrix was used where the columns represented stimuli, either personobjects or lights, and the rows, response categories, either of redness or adjustment. The cell entries were the individual S's responses. For groups with only one $S$ there would be only one entry in any cell, for Group 2 with two individuals there would be two, and so on for each group. Consequently, $T_{x ; y}$, or information transmitted, was for groups and not for individuals except, of course, for the condition I have called Group 1. Analyzing the data in this fashion yielded the results graphically depicted in Fig. 1. Obviously, as size of group increased $T_{x ; y}$ decreased. This was true for both classes of stimuli. The Spearman rank order

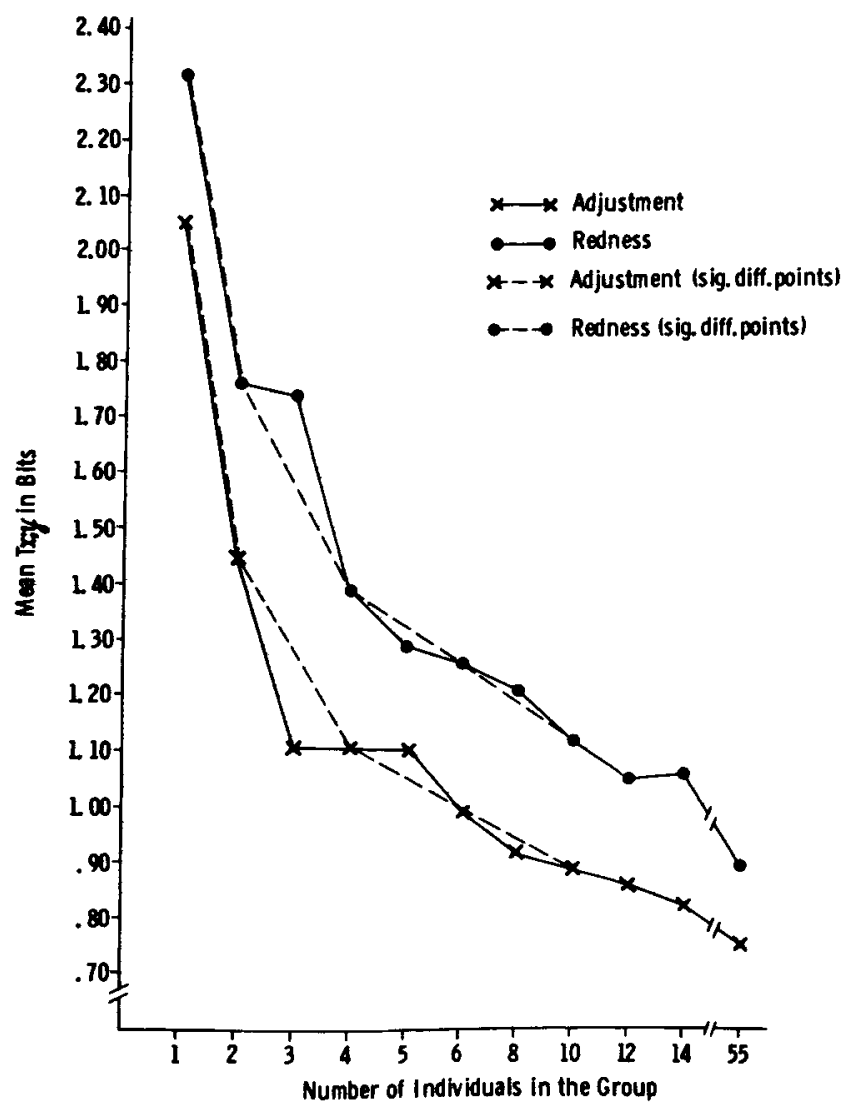

Fig. 1. Mean group $T_{x: y}$ for both tasks as a function of group size (statistically sig. points indicated by dashed lines). 


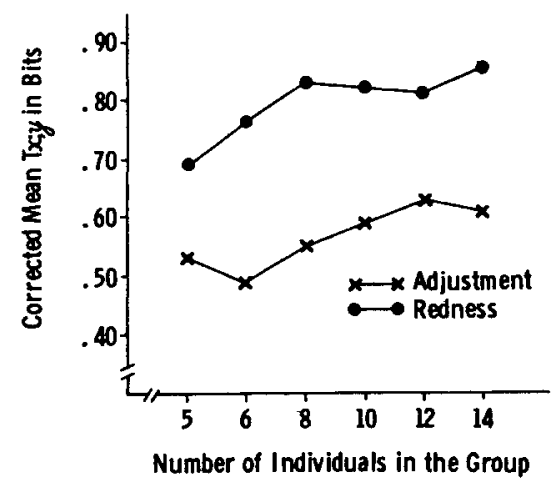

Fig. 2 Corrected mean $T_{x: y}$ for both tasks as a function of group size.

correlation for group size and average $T_{x} ; y$ for judgments of adjustment was 1.00 , and for redness was .98 .

The data, i.e., $T_{x: y}$ values, for the two tasks were further analyzed by an analysis of variance. Group size and type of task were found to significantly $(p<.01)$ affect amount of information transmitted, Fs of 63.11 and 84.36 , respectively. More information was transmitted judging redness of lights than in judging adjustment from written statements. Also, group size significantly affected the amount of information transmitted by a group, as the group got larger information decreased. Tukey's w-procedure (Steel \& Torrie, 1960) indicated that $T_{x ; y}$ was significantly ( $p<.01$ ) different for Groups 1 and 3,3 and 5 , and 5 and 12. This is graphically depicted by the dashed lines in Fig. 1 .

Miller \& Madow (1954) suggested that $\mathrm{T}_{\mathbf{x} ; \mathbf{y}}$ computed in the above fashion for individuals was an over-estimate and that an unbiased estimate could be obtained by subtracting a correction factor, the correction factor being $\mathrm{df} / 1.3863 \mathrm{n}$; where df was $(x-1)(y-1), x$ being the number of judgmental categories and $y$ being number of response categories, or for the present data $5 \times 5$ or 25 , and $\mathrm{n}$ being the total number of observations, or for the present data the number of stimuli by the number of Ss. The corrected $T_{x ; y}$ values are plotted in Fig. 2 and it can be observed that this does not eliminate but tends to reverse the group size effect. However, it was not clear if these corrected figures were "truer" than the uncorrected ones. Attneave (1959) mentioned that correction should only be used where (1) $n>$ df and (2) where there were few, or ideally no, zero cell frequencies. For all groups, Condition 2 was violated, and for Groups 1.4 so was Condition 1. Consequently, it was not clear if correction was appropriate.

Discussion. In discussing these results it was necessary to separate their, perhaps, psychological explanation from their implications for uncertainty analysis.

Statistical Considerations. It was clear that uncertainty for groups was different than that for individuals, at least when stimulus and response uncertainty was relatively high. In groups, the joint occurrence of stimuli and responses $\left(\mathrm{H}_{\mathbf{x} ; \mathbf{y}}\right)$ was more uncertain than for individuals and was reflected in lowered $T_{x}$; for groups. As group size increased, $H_{x: y}$ increased reducing $T_{x ; y}$.
From the data the exact nature of this function was unknown but it was suggested that as group size approximately doubled, up to an unknown point, a significant decrease in $T_{x ; y}$ would obtain.

On the surface, it appeared that adjusting for bias, as suggested by Miller \& Madow (1954), might eliminate the group size effect. However, the limiting conditions mentioned above for the use of the correction for bias were so restrictive as to make its applicability to the data suspect. At any rate, when the correction procedure was applied it tended to reverse the group size effect rather than eliminate it. In other words, a slightly positive relationship between group size and $T_{x ; y}$ was found. Admittedly the data did not meet the assumptions required for correction. In fact, the number of cell entries with zeros increased as group size decreased. This exactly paralleled the positive relationship obtained between group size and adjusted $T_{x: y}$, suggesting that as the number of zero cells increased (resulting in actually fewer of than the 25 on which the calculations for the data were based) the "unbiased" estimate tended to underestimate the actual $T_{x ; y}$. Moreover, changes in $T_{x ; y}$ were obviously due to changes in $H_{x ; y}$ and correction for bias does not deal with this fact. Thus, further suggesting that this correction was not applicable for eliminating the group size effect.

It was concluded from this that, at present, there was no satisfactory way for correcting for bias of Group $T_{x ; y}$ for small groups (if there be such), and that the adjustment procedure provided by Miller \& Madow (1954) would not eliminate the group size effect; indeed it tended to reverse its direction.

Psychological Considerations. A psychological explanation of the data was not hard to come by. For the stimuli and responses used, average information transmitted was relatively high, 2.047 for adjustment and 2.314 for redness. However, for groups, fewer reliable discriminations were possible suggesting that the individuals composing a group did not agree among themselves, and as group members were added what agreement there was declined further. Put another way, groups possess a lot of noise which increased as group size increased. It should be added that this was not exactly a linear function whereby adding one individual to a group resulted in a certain increase in noise. It also appeared that this phenomenon was limited to relatively small groups.

\section{REFERENCES}

ATTNEAVE, F. Applications of information theory to psychology. New York: Henry Holt and Co., Inc., 1959. P. 66.

BIERI, J., ATKINS, A. L., BRIAR, S., LEAMAN, R. L., MILLER, H., \& TRIPODI, T. Clinical and social judgment: the discrimination of behavioral information. New York: John Wiley \& Sons, Inc., 1966.

CLEMENT, D. E. Uncertainty and latency of verbal naming responses as correlates of pattern goodness. J. verbal Leam. verbal Behav., 1964, 3, 150-157.

MILLER, G. A., \& MADOW, W. G. On the maximum likelihood estimate of the Shannon-Wiener measure of information. Air Force Cambridge Research Center: Technical Report 54-75, August, 1954.

STEEL, R. G. D., \& TORRIE, J. H. Principles and procedures of statistics. New York: McGraw-Hill Book Co., Inc., 1960. P. 109.

$$
\text { NOTE }
$$

1. Published by Educational and Industrial Testing Service: Copyright 1959 by H. J. Eysenck. 